\title{
Vortices in a mesoscopic cone: A superconducting tip in the presence of an applied field
}

\author{
Yajiang Chen, ${ }^{1}$ Mauro M. Doria, ${ }^{1,2}$ and F. M. Peeters ${ }^{1, *}$ \\ ${ }^{1}$ Departement Fysica, Universiteit Antwerpen, Groenenborgerlaan 171, B-2020 Antwerpen, Belgium \\ ${ }^{2}$ Instituto de Física, Universidade Federal do Rio de Janeiro, 21941-972 Rio de Janeiro, Rio de Janeiro, Brazil
}

(Received 26 November 2007; published 25 February 2008)

\begin{abstract}
A mesoscopic superconductor in the shape of a circular cone, and size of the order of the coherence length, is investigated theoretically. The external magnetic field is applied perpendicular to its circular basis, and vortex states are obtained in the framework of the Ginzburg-Landau theory. We find patterns made of giant vortex states (GVS), curved multivortex states (MVS), and a combination of both of them. The results are summarized in phase diagrams, where the GVS and MVS regimes are determined according to the applied field and geometric parameters. We find that superconductivity persists up to fields much larger than the upper critical field $\left(H_{c 2}\right)$ in case of a very small apex angle. The results are relevant to understand the properties of superconducting tips, which are currently used in scanning tunneling microscopy, in the presence of an external applied field.
\end{abstract}

DOI: 10.1103/PhysRevB.77.054511

PACS number(s): 74.78.Na, 74.25.Dw, 68.37.Ef, 74.50. $+\mathrm{r}$

\section{INTRODUCTION}

The importance of the study of superconducting mesoscopic structures is increasing because of the availability of modern techniques that enable their fabrication under controlled conditions. Mesoscopic structures are those with dimensions comparable to the coherence length $\xi$ or to the magnetic penetration depth $\lambda$. The mesoscopic size makes the superconducting properties quite different from those of macroscopic samples, as found in the many geometries experimentally and theoretically studied so far: thin disks, ${ }^{1-7}$ cylinders, ${ }^{8,9}$ rings, ${ }^{3,10,11}$ and spheres. ${ }^{12-14}$ The GinzburgLandau (GL) theory has been proven to give a good account of the properties of mesoscopic superconductors in the whole $H$ - $T$ diagram. ${ }^{15}$

There has been increasing interest to understand the properties of superconducting tips ${ }^{16}$ because of their use in scanning tunneling spectroscopy. The presence of a magnetic field ${ }^{17,18}$ adds an extra tunable parameter to investigate those systems. Recently, Kohen et al. ${ }^{19}$ were able to probe the superfluid velocity on the surface of a superconductor using a superconducting tip. They succeeded in detecting the Doppler shift, which gives information about the vortex cores at $\xi$ scale and also about the supercurrent flow around the vortex within the $\lambda$ scale. Notice that a superconducting tip, and, as a matter of fact, any small protuberance on the surface of a type I superconductor, is always of type II. The situation resembles that of a type I superconducting film that turns into a type II superconductor by its small thickness $(d \sim \xi)$. In this case, the effective London penetration length along the surface becomes equal to $\lambda^{2} / d$. For this reason, tips are expected to remain in the superconducting state in the presence of fields above the thermodynamic type I critical field. . $^{18,20,21}$

The scanning tunneling spectroscopy investigation of surfaces through a superconducting tip in the presence of an applied field must take into account the possible presence of vortices. Previously, Misko et al. modeled theoretically a superconducting bridge with the Ginzburg-Landau theory 22 and studied giant vortex states (GVS). The bridge basically con- sists of two oppositely positioned cones with their apexes in contact. Giant vortices were also found in a mesoscopic wire containing a narrow constriction ${ }^{23}$ as well as the break up of a GVS into a multivortex state (MVS).

The cone can be viewed as a sum over different disks with increasing diameter $D$, and this serves as an extra motivation for its study. For a very thin superconducting disk with diameter $D$ of the order of or less than $2 \xi$, no vortices can enter and only the Meissner state is possible. Above this limit, and for $D<4 \xi$, GVSs are allowed, while for $D>4 \xi$, MVSs are also possible. ${ }^{24}$ Theoretical studies ${ }^{5,15}$ of very thin superconducting disks are in fair agreement with experimental measurements. ${ }^{25,26}$ However, those studies were limited to samples with thickness smaller than $\xi$, with the applied field oriented perpendicularly to the flat geometry. From this aspect, it is clear that the amount of flux penetrating the superconducting cone must be a function of the height, as found here.

GVS and MVS have been recently observed in mesoscopic superconductors. ${ }^{7,27,28}$ Intrinsically novel properties are found, such as induced antivortices that appear for certain values of the applied field ${ }^{29,30}$ in the case of a thin superconducting square. In this paper, we determine the GVS and also the MVS of a single cone with the applied field oriented perpendicularly to the circular basis. We determine the phase diagram that describes the GVS and MVS regimes in the applied field and in the geometric parameter space. The London penetration length is taken much larger than the dimensions of the cone, such that the local magnetic field inside can be taken equal to the applied one. The superconducting order parameter is expressed as a linear expansion of solutions of the linear Ginzburg-Landau equation. We limit the present study to a maximum of two coefficients, which is sufficient to describe the vortex pattern of a small cone such that at most one shell of vortices can be formed. The software packages COMSOL (which is based on the finite element method) and MATLAB are used to solve the linear GL equation for our cone geometry and to calculate the Gibbs free energy of the nonlinear theory.

This paper is organized as follows. In Sec. II, we discuss the theoretical framework used to obtain our results. In Secs. 


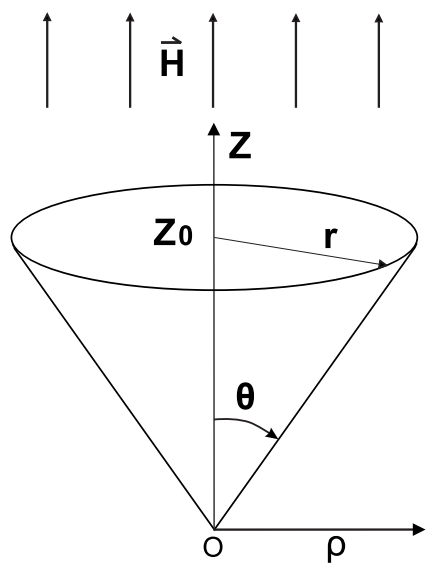

FIG. 1. Coordinate system and the circular cone in the presence of an applied field.

III and IV, we study giant and multivortex states, respectively. In Sec. V, we show the phase diagrams for the cone. Last, in Sec. VI, we draw the major conclusions of our paper. In the Appendix, some solutions of the linearized GL equation, which do not participate in the construction of our physical states, are discussed.

\section{THEORETICAL FRAMEWORK}

The cone geometry is shown in Fig. 1 together with the considered coordinate system. The cone is surrounded by vacuum with an external uniform magnetic field $\vec{H} \equiv H \hat{e}_{z}$ along the major axis. Only two parameters are required to describe a circular cone, among the three existing ones: the height $z_{0}$, the apex angle $\theta$, and the radius $r$. Height, vertex angle, and radius are related by $r=z_{0} \tan (\theta)$, and we choose the first two variables, using the notation $\mathbf{z}_{0^{-}} \theta$, to describe the circular cone.

According to Ginzburg and Landau, the Gibbs free energy of the superconductor near the critical temperature $T_{c}$ can be expanded in powers of the complex order parameter $\Psi(\vec{r})$ that gives the density of Cooper pairs: $|\Psi(\vec{r})|^{2}$. The Gibbs free energy difference between the superconducting state and the normal state is

$$
\begin{aligned}
F_{s}-F_{n}= & \int d V\left\{\alpha(T)|\Psi|^{2}+\frac{1}{2} \beta|\Psi|^{4}\right. \\
& \left.+\frac{\hbar^{2}}{2 m^{*}}\left|\left(\vec{\nabla}-i \frac{2 \pi}{\Phi_{0}} \vec{A}\right) \Psi\right|^{2}-\frac{1}{4 \pi}(\vec{\nabla} \times \vec{A}-\vec{H}) \cdot \vec{H}\right\},
\end{aligned}
$$

with the phenomenological constants $\alpha(T) \equiv \alpha_{0}\left(T-T_{c}\right)<0$, $\beta>0, m^{*}$ (the mass of one Cooper pair), and $\Phi_{0}=h c / 2 e$, the fundamental unit of flux. By minimizing $F_{s}$ with respect to $\Psi(\vec{r})$ and $\vec{A}(\vec{r})$, one obtains the Ginzburg-Landau equations. The following boundary condition is imposed on all external surfaces of the cone:

$$
\left.\vec{n} \cdot\left(\vec{\nabla}-i \frac{2 \pi}{\Phi_{0}} \vec{A}\right) \Psi\right|_{\text {boundary }}=0 .
$$

Because of its small size, we assumed the magnetic field inside and outside the circular cone equal, and so $\vec{\nabla} \times \vec{A}=\vec{H}$. We express quantities in this theory in dimensionless units defined by the following reduced units: the coherence length $\xi(T) \equiv\left\{\hbar^{2} / 2 m^{*}[-\alpha(T)]\right\}^{1 / 2}$ (lengths); $\Phi_{0} / 2 \pi \xi(T)$ (vector potential), and so $H_{c 2}(T)=\Phi_{0} / 2 \pi \xi(T)^{2}$ (magnetic field); $\Psi_{0}$ $=\sqrt{-\alpha(T) / \beta}$ (order parameter); and $F_{0}=\alpha(T)^{2} / 2 \beta$ (free energy). In terms of these dimensionless units, the Gibbs free energy becomes

$$
F=2 \int d V\left\{-|\Psi|^{2}+\frac{1}{2}|\Psi|^{4}+|(\vec{\nabla}-i \vec{A}) \Psi|^{2}\right\},
$$

where we set the normal part of the free energy $F_{n}=0$, and the integration is restricted to the volume of the cone. The boundary condition in dimensionless units becomes

$$
\left.\vec{n} \cdot(\vec{\nabla}-i \vec{A}) \Psi\right|_{\text {boundary }}=0 .
$$

Following Ref. 6, we introduce the $\hat{L}$ linear operator, in terms of which the nonlinear GL equation becomes

$$
\hat{L} \psi=-|\psi|^{2} \psi, \quad \hat{L} \equiv-(\vec{\nabla}-i \vec{A})^{2}-1 .
$$

Consequently, only the negative eigenvalues $\Lambda$ of this $\hat{L}$ correspond to the superconducting state.

We introduce cylindrical coordinates $(\rho, \varphi, z)$ into the problem because the cone has symmetry around the azimuthal angle $\varphi$. The external magnetic field, $\vec{H}=H \hat{e}_{z}$, is obtained from $\vec{A}=(H \rho / 2) \overrightarrow{e_{\varphi}}$, which satisfies the London gauge $\nabla \cdot \vec{A}=0$. The order parameter is taken of the form

$$
\Psi(\rho, \varphi, z)=\psi_{L}(\rho, z) e^{i L \varphi},
$$

where the angular momentum quantum number $L$ is an integer due to the single valuedness of the order parameter. The following linear equation must be solved:

$$
\hat{L} \psi_{L, n}(\rho, z)=\Lambda_{L, n} \psi_{L, n}(\rho, z),
$$

where $n(=0,1,2,3, \ldots)$ enumerates the different states at a certain angular momentum quantum number $L$. Following Refs. 1 and 2, we restrict ourselves to $n=0$ because these states are known to give the major contribution, and hereafter drop the $n$ in $\psi_{L, n}$ in favor of a short notation, such that $\psi_{L}$ stands for $\psi_{L, n=0}$. Similarly, $\Lambda_{L}$ is short for $\Lambda_{L, n=0}$. A study of some of the $n \neq 0$ eigenvalues is given in the Appendix. This operator in dimensionless units and using cylindrical coordinates becomes

$$
\hat{L}=-\frac{1}{\rho} \frac{\partial}{\partial \rho}\left(\rho \frac{\partial \Psi}{\partial \rho}\right)-\frac{\partial^{2} \Psi}{\partial z^{2}}+\frac{1}{\rho^{2}}\left(L-\frac{1}{2} H \rho^{2}\right)^{2}-1 .
$$

Then a general expression of the order parameter, expressed in this basis, with maximum angular momenta $L$, is 


$$
\Psi(\rho, \varphi, z)=\sum_{L_{j}=0}^{L} C_{L_{j}} \psi_{L_{j}}(\rho, z) \exp \left(i L_{j} \varphi\right) .
$$

Substituting Eq. (9) into the free energy expression of Eq. (3) gives $F$ as a function of the complex coefficients $\left\{C_{L_{j}}\right\}$. To obtain the equilibrium vortex configurations, $F$ must be minimized with respect to the linear solution free parameters $\left\{C_{L_{j}}\right\}$,

$$
\frac{\partial F}{\partial C_{L j}}=0 \quad \text { and } \quad \frac{\partial F}{\partial C_{L_{j}}^{*}}=0,
$$

and the stability of the solution verified. If the state is stable, then its Hessian matrix,

$$
M_{\text {Hesse }}=\left(\begin{array}{cccc}
\frac{\partial^{2} F}{\partial C_{0}^{2}} & \frac{\partial^{2} F}{\partial C_{0} \partial C_{1}} & \cdots & \frac{\partial^{2} F}{\partial C_{0} \partial C_{L}^{*}} \\
\frac{\partial^{2} F}{\partial C_{0} \partial C_{1}} & \frac{\partial^{2} F}{\partial C_{1}^{2}} & \cdots & \frac{\partial^{2} F}{\partial C_{1} \partial C_{L}^{*}} \\
\cdots & \ldots & \cdots & \ldots \\
\frac{\partial^{2} F}{\partial C_{L}^{*} \partial C_{0}} & \frac{\partial^{2} F}{\partial C_{L}^{*} \partial C_{1}} & \cdots & \frac{\partial^{2} F}{\partial C_{L}^{* 2}}
\end{array}\right),
$$

should be positive definite. The search for both GVS and MVS relies on the computation of the following integrals for the eigenvectors of Eq. (7):

$$
\begin{gathered}
A_{L_{1}, L_{2}}=\int_{0}^{z_{0}} d z \int_{0}^{z \tan (\theta)} \rho d \rho \psi_{L_{1}}^{2}(\rho, z) \psi_{L_{2}}^{2}(\rho, z), \\
A_{L_{i}}=\int_{0}^{z_{0}} d z \int_{0}^{z \tan (\theta)} \rho d \rho \psi_{L_{i}}^{4}(\rho, z), \\
B_{L_{i}}=\int_{0}^{z_{0}} d z \int_{0}^{z \tan (\theta)} \rho d \rho \psi_{L_{i}}^{2}(\rho, z) .
\end{gathered}
$$

In case that just a single term is considered in Eq. (9), only the GVS states can be described by

$$
\Psi(\rho, \varphi, z)=C_{L} \psi_{L}(\rho, z) e^{i L \varphi},
$$

where the coefficient $C_{L}$ is a constant to be determined by imposing on the free energy [Eq. (3)] the conditions of Eqs. (10) and (11). One obtains

$$
C_{L}= \pm \sqrt{-\Lambda_{L} \frac{B_{L}}{A_{L}}}
$$

and for the free energy,

$$
F=-2 \pi \Lambda_{L}^{2} \frac{B_{L}^{2}}{A_{L}} .
$$

Inspection of the second derivative of the free energy shows that a negative eigenvalue $\Lambda_{L}$ is the condition to render the state stable.

MVS can be constructed from a two-component order parameter in Eq. (9). In this situation too, analytical expres- sions for the coefficients, the free energy, and the stability conditions can be obtained. The two-component order parameter is

$$
\Psi(\rho, \varphi, z)=C_{L_{1}} \psi_{L_{1}}(\rho, z) e^{i L_{1} \varphi}+C_{L_{2}} \psi_{L_{2}}(\rho, z) e^{i L_{2} \varphi} .
$$

In case one of the coefficients $\left(C_{L_{1}}, C_{L_{2}}\right)$ vanishes, then this state becomes a GVS. If these coefficients are nonzero, then this is a MVS. If both of them are zero, then the normal state is reached. The description of a GVS through two components is relevant to check its stability with respect to the MVS. According to Ref. 1, whether $C_{L_{i}}$ are real or complex does not affect the value of the free energy, so we can regard them as real. By minimizing the Gibbs free energy with respect to $C_{L_{1}}$ and $C_{L_{2}}$ using Eq. (10), we obtain the possible equilibrium states: (i) The normal state with $\Psi(\rho, \varphi, z)=0$ corresponds to $C_{L_{1}}=C_{L_{2}}=0$. (ii) The GVS with $C_{L_{2}}=0$ is $\Psi(\rho, \varphi, z)=C_{L_{1}} \psi_{L_{1}} e^{i L_{1}^{2} \varphi}$, with $C_{L_{1}}=\left(-\Lambda_{L_{1}} B_{L_{1}} / A_{L_{1}}\right)^{1 / 2}$; the $C_{L_{1}}=0$ state is $\Psi(\rho, \varphi, z)=C_{L_{2}} \psi_{L_{2}} e^{i L_{2} \varphi}$, with $C_{L_{2}}$ $=\left(-\Lambda_{L_{2}} B_{L_{2}} / A_{L_{2}}\right)^{1 / 2}$. (iii) The MVS of Eq. (18) has the coefficients

$$
\begin{aligned}
& C_{L_{1}}= \pm\left(\frac{\Lambda_{L_{1}} A_{L_{2}} B_{L_{1}}-2 \Lambda_{L_{2}} A_{L_{1}, L_{2}} B_{L_{2}}}{-A_{L_{1}} A_{L_{2}}+4 A_{L_{1}, L_{2}}^{2}}\right)^{1 / 2}, \\
& C_{L_{2}}= \pm\left(\frac{\Lambda_{L_{2}} A_{L_{1}} B_{L_{2}}-2 \Lambda_{L_{1}} A_{L_{1}, L_{2}} B_{L_{1}}}{-A_{L_{1}} A_{L_{2}}+4 A_{L_{1}, L_{2}}^{2}}\right)^{1 / 2} .
\end{aligned}
$$

The free energy of the GVS is

$$
F_{L_{i}}=-2 \pi \Lambda_{L_{i}}^{2} \frac{B_{L_{i}}^{2}}{A_{L_{i}}}, \quad i=1,2,
$$

and that of the MVS,

$$
\begin{aligned}
F_{L_{1}, L_{2}}= & 2 \pi \frac{1}{A_{L_{1}} A_{L_{2}}-4 A_{L_{1}, L_{2}}^{2}}\left(-\Lambda_{L_{1}}^{2} A_{L_{2}} B_{L_{1}}^{2}-\Lambda_{L_{2}}^{2} A_{L_{1}} B_{L_{2}}^{2}\right. \\
& \left.+4 \Lambda_{L_{1}} \Lambda_{L_{2}} A_{L_{1}, L_{2}} B_{L_{1}} B_{L_{2}}\right) .
\end{aligned}
$$

We obtain two conditions for the stability of the GVS. For instance, for $C_{L_{1}}=0$ and $C_{L_{2}} \neq 0$, one obtains

$$
\begin{aligned}
-\Lambda_{L_{2}} & >0, \\
-2 \Lambda_{L_{2}} A_{L_{1}, L_{2}} B_{L_{2}} & >-\Lambda_{L_{1}} A_{L_{2}} B_{L_{1}} .
\end{aligned}
$$

For $C_{L_{1}} \neq 0$ and $C_{L_{2}}=0$, the first condition is $L_{1}>0$, and the second one is obtained from the above one through the interchange $L_{1} \leftrightarrow L_{2}$. There are three conditions that must be fulfilled to render the MVS stable, namely,

$$
\begin{gathered}
\Lambda_{L_{1}} A_{L_{2}} B_{L_{1}}-2 \Lambda_{L_{2}} A_{L_{1}, L_{2}} B_{L_{2}}>0, \\
\Lambda_{L_{2}} A_{L_{2}} B_{L_{1}}-2 \Lambda_{L_{2}} A_{L_{1}, L_{2}} B_{L_{2}}>0, \\
4 A_{L_{1}, L_{2}}-A_{L_{1}} A_{L_{2}}>0 .
\end{gathered}
$$



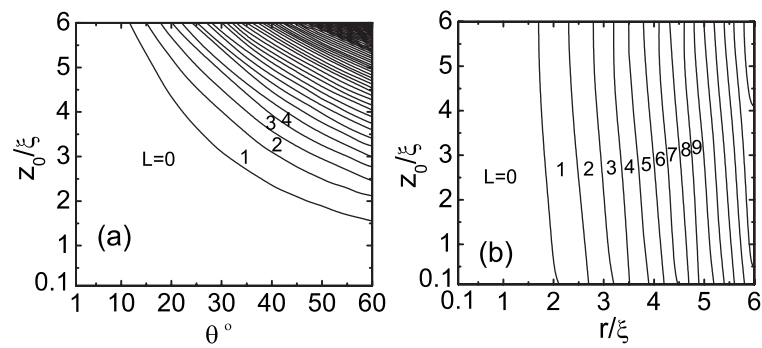

FIG. 2. The largest vorticity that can nucleate in the circular cone.

\section{GIANT VORTEX STATES}

In this section, we numerically solve Eq. (7) under the appropriate boundary condition of Eq. (4), applied to the external surfaces of the cone. In Fig. 2, we describe the obtained maximum vorticity in two different ways in terms of the cone parameters. The maximum vorticity corresponds to the GVS with the highest angular momentum quantum number $L$ able to render a negative eigenvalue for $\hat{L}$. Figure 2(a) shows the height versus apex angle diagram with the maximum vorticity, limited to the $0.1 \xi-6 \xi$ and to the $1^{\circ}-60^{\circ}$ ranges, respectively. The maximum vorticity is also given in Fig. 2(b) as height versus radius, this last ranging from $0.1 \xi$ to $6 \xi$. From these maximum vorticity plots, we learn that keeping the circular basis $(r)$ fixed and increasing the height $\left(z_{0}\right)$ do not change significantly the maximum vorticity as much as it does by increasing the circular basis $(r)$ under fixed height $\left(z_{0}\right)$. In this last case, a substantial increase of the maximum vorticity is possible. Notice that for the chosen ranges of Fig. 2, the cones with $z_{0} \leqslant 1.5 \xi$, or $\theta \leqslant 12^{\circ}$, or $r$ $\leqslant 1.7 \xi$ cannot sustain a vortex state.

Next we show the eigenvalue versus magnetic field and the free energy versus magnetic field diagrams, some Cooper-pair density, and phase distributions of the following cones:

(i) $2.5-30\left(z_{0}=2.5 \xi, \theta=30^{\circ}, r=1.44 \xi\right)$,

(ii) $2.5-45\left(z_{0}=2.5 \xi, \theta=45^{\circ}, r=2.5 \xi\right)$,

(iii) $4.0-45\left(z_{0}=4.0 \xi, \theta=45^{\circ}, r=4 \xi\right)$,

(iv) $2.5-60\left(z_{0}=2.5 \xi, \theta=60^{\circ}, r=4.33 \xi\right)$.

According to Fig. 3, the maximum vorticity for these cones is the following: $L=0$ for $2.5-30, L=2$ for $2.5-45, L$ $=6$ for 4.0-45, and $L=7$ for 2.5-60, respectively.

Figures 3(e)-3(h), obtained from Eq. (17), show that for a certain $H$ there may exist more than one stable GVS. The lowest one in free energy is the ground state, and the other ones are metastable states. The dotted horizontal line is a guide for the eye to indicate the normal state. Increasing $H$ causes the ground state to transit to a higher vorticity, $L$ $\rightarrow L+1$. We observe that the crossing points, defined by the intersection between $L$ and $L+1$ curves, shift toward lower magnetic fields as the cone sustains higher vorticities. Also, notice that the maximum field able to destroy the superconducting state rapidly shifts toward $H_{c 2}$ as the maximum cone vorticity increases. These results are similar to those found in Ref. 13 for mesoscopic superconducting spheres. Next we investigate the Cooper-pair density of the different GVS, ob-
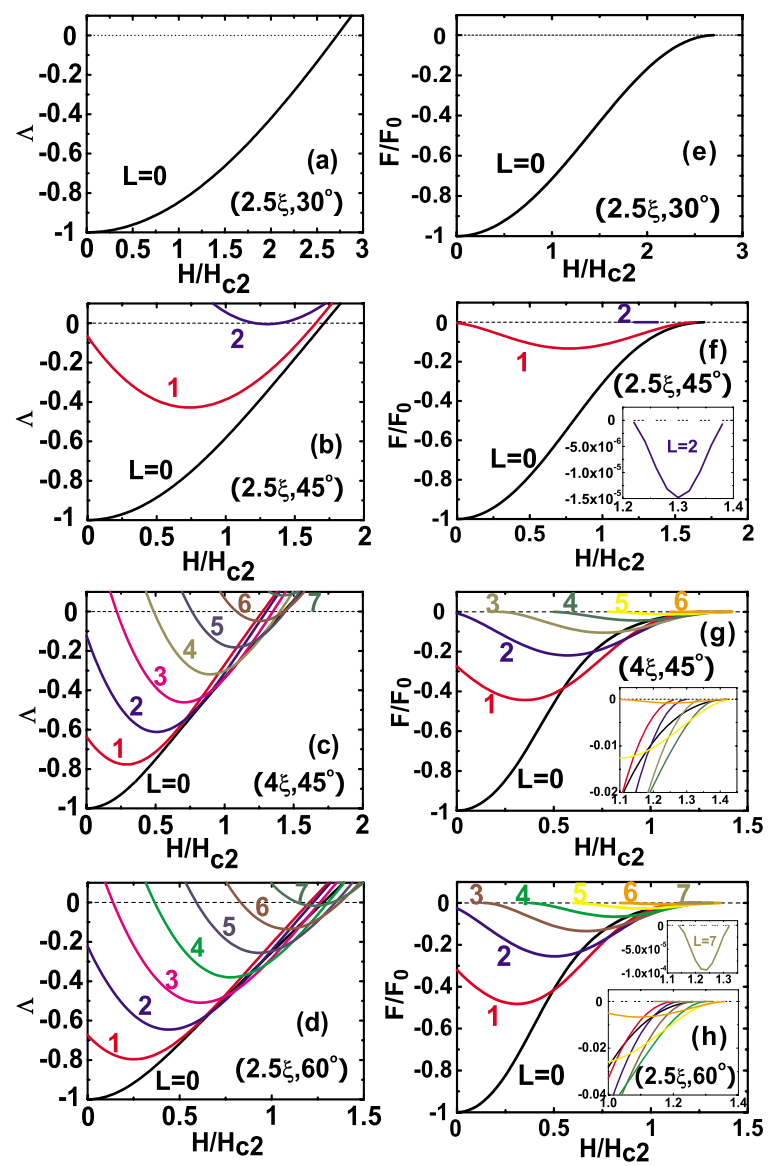

FIG. 3. (Color online) Eigenvalue and free energy vs applied magnetic field plots for different circular cones. The insets are enlargements of some of the curves in a limited $H$ region.

tained from Eq. (15): $|\Psi(\rho, \varphi, z)|^{2}=-\Lambda_{L}\left(B_{L} / A_{L}\right) \psi_{L}(\rho, z)^{2}$.

Figure 4 shows a contour plot of the GVS Cooper-pair density for the 2.5-45 cone in the cross section $\rho-z$ plane for some selected vorticities and applied magnetic field values.

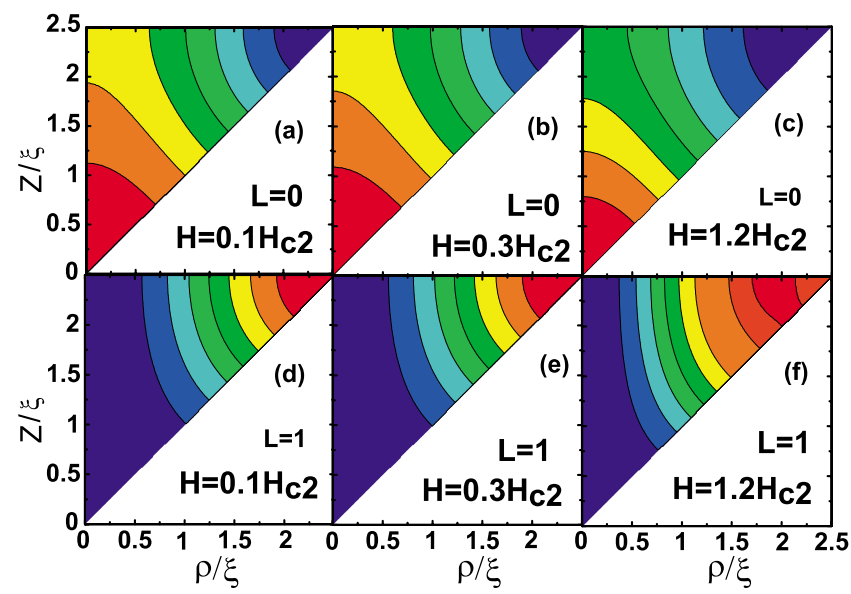

FIG. 4. (Color online) The Cooper-pair density in the cross section $\rho-z$ plane for the $2.5-45$ cone. Selected $L$ and $H$ values are shown here. Blue to red means density range from minimum to maximum. 

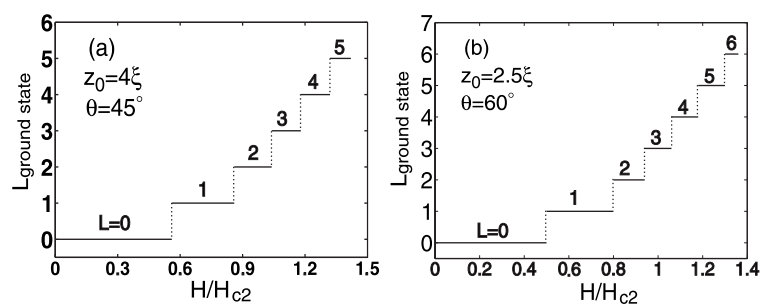

FIG. 5. The vorticity of the ground state vs the applied magnetic field for two selected cones.

For this cone, although there are three possible vorticities available, the ground state is always the zero vortex state $(L=0)$ for any $H$, according to Fig. 3(f). The density for this small cone is dominated by surface superconductivity that controls the state within the depth of $\xi$ from the surface (see Ref. 31). In Figs. 4(a)-4(c), $|\Psi|^{2}$ is found to be maximum at the tip because of the enhanced surface superconductivity in small regions (see, e.g., Ref. 31 for the two-dimensional case). Increasing the cone size rapidly increases the number of different ground states, as shown in Fig. 5 for the 4.0-45 and 2.5-60 cones.

Figure 6 displays the GVS Cooper-pair density at the $\rho-z$ cross section for the 4.0-45 cone, for selected $L$ and $H$ values. According to Fig. 5, the densities of Figs. 6(a), 6(e), and 6(i) correspond to ground states.

From Fig. 6, we can draw some general conclusions about the GVS in the cone. The zero vortex $(L=0)$ state reaches maximum (red) density around the symmetry axis near the apex. There the cone reaches dimensions comparable to $\xi$ and surface effects dominate the physics. The growth of the

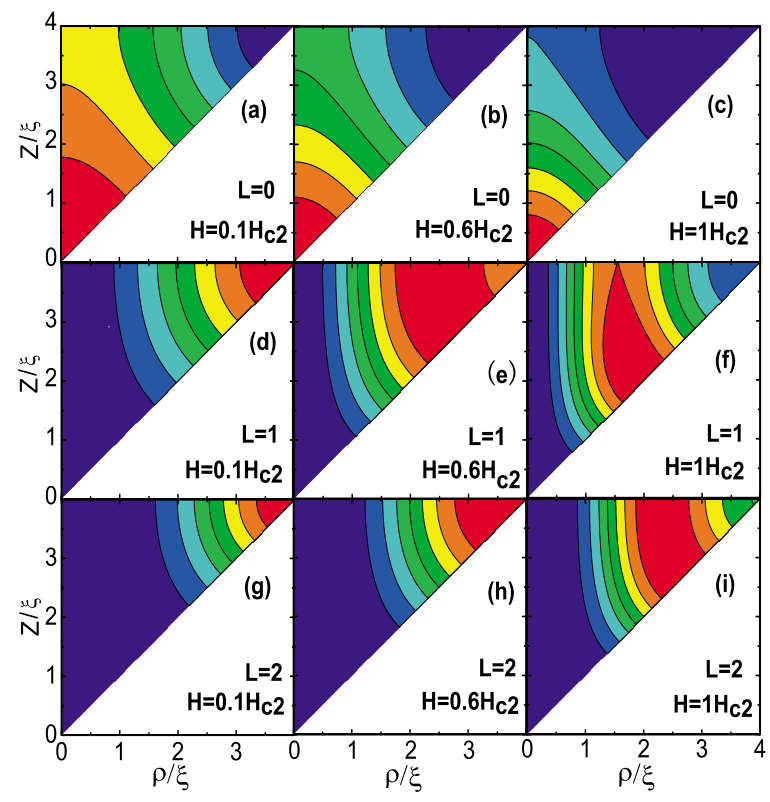

FIG. 6. (Color online) The Cooper-pair density is shown in the $\rho-z$ cross section of the cone 4.0-45 for the following magnetic fields and vorticities: $H / H_{c 2}=0.1,0.6,1.0$; [(a)-(c) $] L=0$, [(d)-(f)] $L=1$, and $[(\mathrm{g})-(\mathrm{i})] L=2$. Blue to red means density range from minimum to maximum.

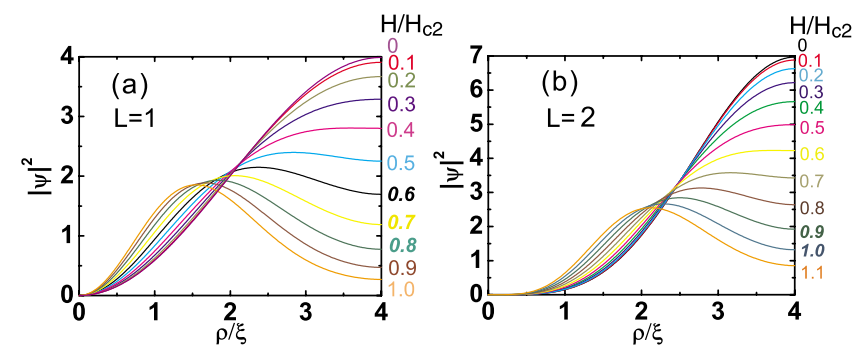

FIG. 7. (Color online) The Cooper-pair density of giant vortex states (a) $L=1$ and (b) $L=2$ at the $z=4 \xi$ plane for the circular cone 4.0-45. According to Fig. 5, the profiles of curves $\mathrm{H} / \mathrm{H}_{c 2}$ $=0.6,0.7,0.8$ in (a), and of curves $H / H_{c 2}=0.9,1.0$ in (b), are ground state densities.

minimum (blue) density at the edge of the circular basis for fixed $L$ and increasing $H$ in Fig. 6 suggests that vortex entrance will take place there $\left[(z, \rho) \sim\left(z_{0}, z_{0} \tan (\theta)\right)\right]$. For instance, this suggests that states represented by Figs. 6(b) and 6(c) cannot be the ground state. Indeed, for $L=1$, a vortex takes over the center of the cone, and consequently, the density reaches a minimum there. Again the depletion of the density near the edge of the circular basis is seen in Fig. 6(f), and taken again as indicative that this state is not fit to be a ground state. For $L=2$, the ground state corresponds to Fig. 6(i). This state also presents a small depletion near the edge of the circular basis, interpreted here as a signal of a weaker stability and, consequently, of an approaching matching field that will eventually give rise to a higher angular momentum state.

To get further insight into the GVS Cooper-pair density, we plot it versus $r$ along the circular basis $\left(z=z_{0}\right)$ for several $H$ values in Fig. 7. The density profiles are shown for the $L=1$ and $L=2$ states of the 4.0-45 cone, and resemble similar results previously obtained for the thin disk (see Fig. 2 of Ref. 6).

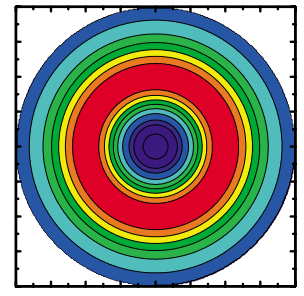

(a)

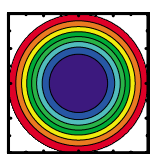

(b)

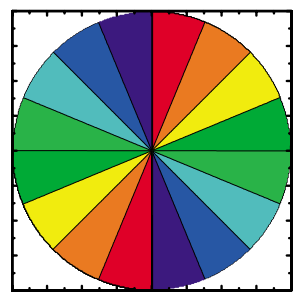

(c)

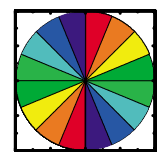

(d)
FIG. 8. (Color online) The Cooper-pair density and the phase of order parameter in two cross section planes, $z=z_{0}$ and $z=z_{0} / 2$, are shown for the 4.0-45 core at the applied magnetic field $H=1.2 H_{c 2}$ with vorticity $L=2$. Blue to red means density range from minimum to maximum for all plots; however, with different meanings. Blue to red for (a) and (b) means maximum to minimum density, whereas for (c) and (d), it indicates $0-2 \pi$ range. 


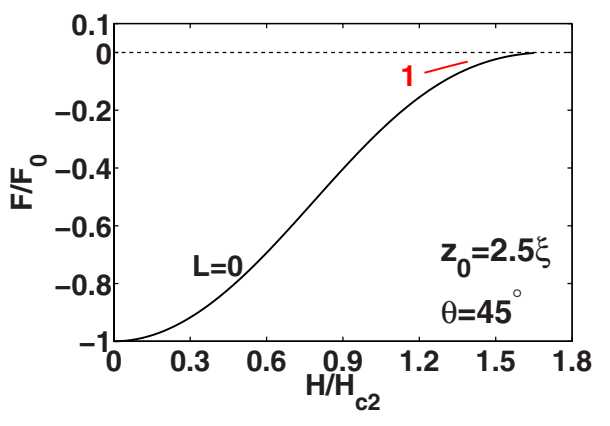

FIG. 9. (Color online) The free energy of the 2.5-45 cone as a function of the applied magnetic field. These $L$ giant vortex curves are those of Fig. 3(f), but subjected to distinct stability conditions.

The phase of the order parameter is an important tool to determine the vorticity because it is equal to $L \times \varphi$ at any $(\rho, \varphi, z)$ point inside the cone. ${ }^{32}$ Thus a GVS with vorticity $L$ is identified by the presence of $L$ consecutive red to blue hues. Figure 8 provides an example as it shows the density and the phase at two cross section planes of the 4.0-45 cone, taken at the circular basis and at the middle $(L=2$ and $H$ $=1.2 H_{c 2}$ ) of the cone.

An important remark concerns the two ways to treat the GVS, either as a one- or a two-component order parameter, provided that in the last approach one of the components is taken equal to zero. Obviously, they lead to the same order parameter and free energy. However, their stability condition is distinct. Within the single component description, it suffices that the eigenvalue is negative to guarantee the stability, whereas for the two-component wave function, there is an extra condition given in Eq. (22). For this reason, we display the free energy of the GVS for the 2.5-45 cone in Fig. 9, which has already been plotted in Fig. 3(f). The largest vorticity curve $(L=2)$, already presented in Fig. 3(f), has totally disappeared here and the $L=1$ curve is substantially reduced in comparison to Fig. 3(f). The zero vortex regime $(L=0)$ is the same for both plots.

\section{MULTIPLE VORTEX STATES}

Here, we study the two-component order parameter of Eq. (18), which is able to describe both GVS and MVS. The two-component approximation is good in case there are vortices in the center and at most in one shell around this center, an approximation suitable for small mesoscopic superconductors. We use the notation $\left(L_{1}, L_{2}\right)$ to indicate the MVS whose order parameter is formed by $\psi_{L_{1}}$ and $\psi_{L_{2}}$, and $L$ for the giant vortex state with vorticity $L$.

The stability of GVS and MVS states is based on Eqs. (22) and (23), respectively. For the 4.0-45 cone, the following states are stable according to these conditions: $L, L$ $=0-6(\mathrm{GVS}) ;(0, L), L=2-6(\mathrm{MVS}) ;(1, L), L=4-6(\mathrm{MVS})$; and $(2, L), L=6$ (MVS). Figure 10 shows the free energy of several states of the 4.0-45 cone, as a function of $H$. Figure 10(b) shows a zoom of the high $H$ region. Notice that the GVS lines superposed by MVS lines are not visible. The GVS curves are solid lines, whereas the MVS curves are
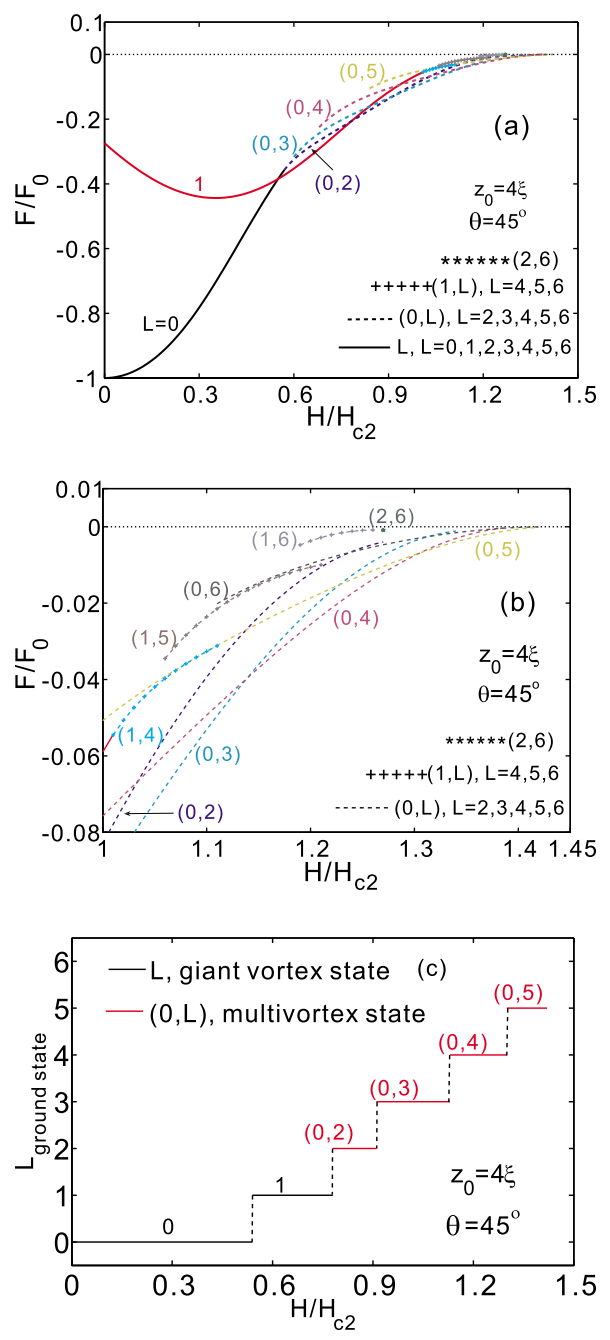

FIG. 10. (Color online) The free energy of the 4.0-45 cone as a function of the applied magnetic field, and (b) is a zoom of the high field region of (a). Solid curves are GVS and the others are different MVSs. (c) is the vorticity of the ground state as a function of the applied magnetic field.

represented either by a dashed or a dotted line. The horizontal dotted line is a guide for the eye for the $F=0$ line. The MVS with vorticity $L=4$ has the following free energy ordering: the $(0,4)$ state is lower than the $(1,4)$ state in their common magnetic field region, and the $(1,4)$ state is lower than the $(2,4)$ state in their common magnetic field region. Similarly, for the MVS with vorticity $L=5$, we observe that the $(0,5)$ state is lower than the $(1,5)$ state, which in turn is lower than the $(2,5)$ state. Curiously, we do not find any intersect (matching fields) between MVS with the same vorticity, and intersects are found only between curves with different vorticities. In Fig. 10(c), we show the vorticity of the ground state for this 4.0-45 cone as a function of $H$, and find that the vorticity of the ground state goes from 0 to 5 for increasing field. The ground state is mixed by the giant vortex states $(L=0-5)$ and the MVS $(0, L)$, where $L=2-5$. There are no GVS $(L=6)$ and other MVS existing in the ground state. Notice that near the superconducting/normal boundary, we have a MVS, while in a superconducting disk, this is usually a giant vortex state. 

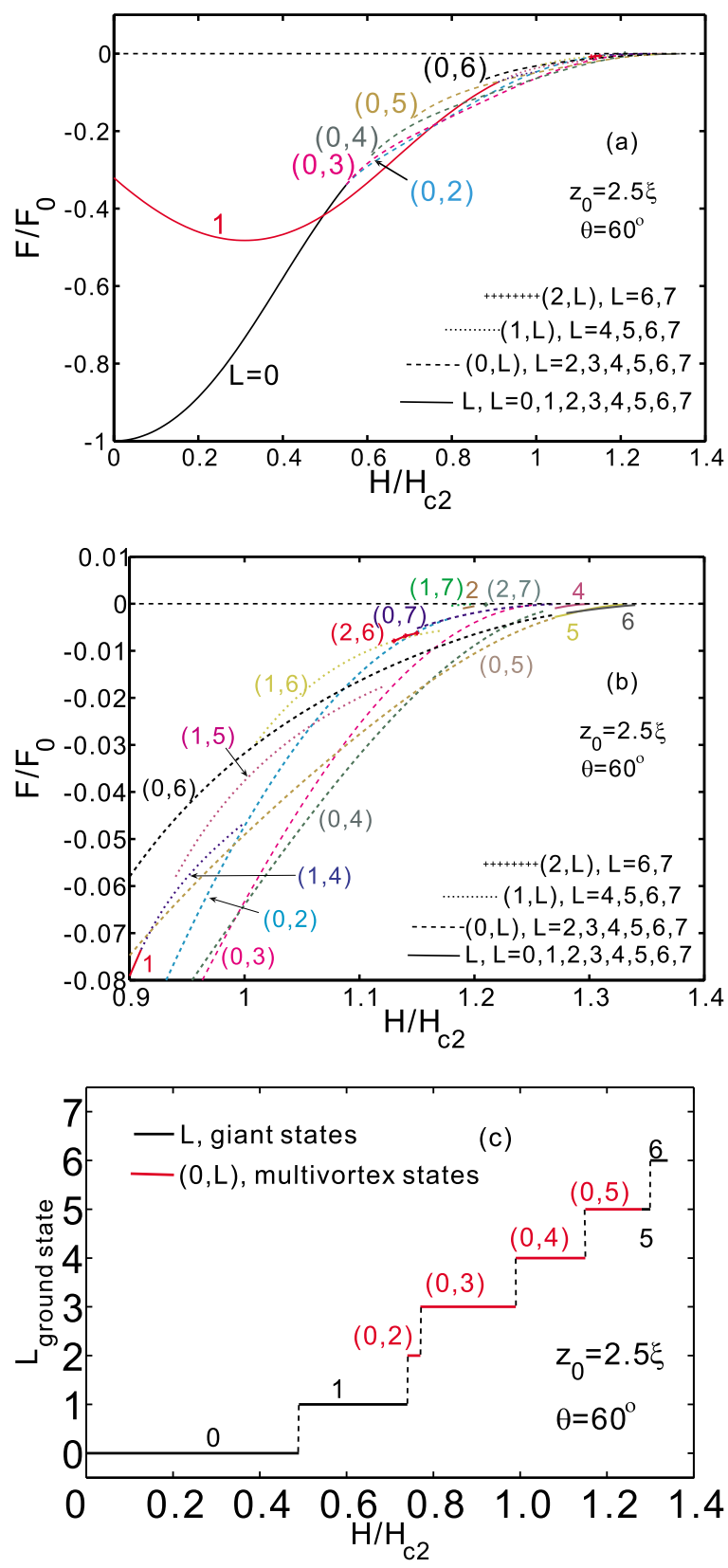

FIG. 11. (Color online) The free energy of the 2.5-60 cone as a function of the applied magnetic field, (b) is a zoom of the high field region of (a), and (c) is the vorticity of the ground state as a function of the applied magnetic field.

Next we repeat the same kind of analysis for the 2.5-60 cone. Figure 11 shows the free energy as a function of $H$, where Fig. 11(b) is a zoom of the high $H$ region. The regions of the GVS curves that overlap with the MVS curves are not visible in this figure. The curves are represented in the following way: $L$ GVS by solid curves, the $(1, L)$ states by dashed curves with a star mark, and the $(2, L)$ states by dashed curves with a plus mark. We find the following states to be stable: GVS with $L=0-7$; MVS $(0, L), L=2-7 ;(1, L)$, $L=4-7$; and $(2, L), L=6-7$. Now, we focus on some MVSs which share the same $L$ vorticity. The states $(0,6),(1,6)$, and $(2,6)$ have vorticity 6 , and within their common magnetic field region, the $(0,6)$ state is the lowest in free energy, while the $(2,6)$ is the highest. Figure 11(b) shows that the states $(0,6)$ and $(1,6)$ have the same free energy at $H / H_{c 2}=1.01$, which is also the case for the states $(1,6)$ and $(2,6)$ at $H / H_{c 2}=1.13$. The $(0, L)$ state has lower energy than the $(1, L)$ state in their common magnetic field region for $L=4$, 5 , and 7 . There is no common field region for states $(1,7)$ and $(2,7)$. Figure $11(\mathrm{c})$ shows that the ground state vorticity for the 2.5-60 cone runs from 0 to 6 as a function of increasing $H$. The ground state is mixed by the GVS $(L=0-6)$ and the MVS $(0, L)$, where $L=2-5$. There are no GVS $(L=7)$ and other MVS nucleating as ground state. Comparison between Figs. 11(c) and 10(c) show interesting differences between these two cones. For instance, the field region for the stability of the $(0,2)$ ground state is much smaller in the former case than in the latter.

Next we study the Cooper-pair density and the phase of the order parameter in several situations. Figures 12 and 13 show several plots for the 4.0-45 cone, and their main difference is that Fig. 13 contains a vortex in the center, whereas Fig. 12 does not. By increasing the field, the following features are observed, where $L$ is the total vorticity. For the $(0, L)(L=2-6)$ states, there are $L$ single vortices. They are arranged on a shell with no vortex in the center. The Cooperpair density is maximum at the apex. For the $(1, L)(L$ $=4-6)$ states, there are $L$ vortices with $(L-1)$ forming a shell and a vortex in the center. For the $(2,6)$ state, the number of vortices is 5 , while the vorticity is 6 . Four of them are located on a shell with a giant vortex in the center with vorticity 2.

The cone displays the interesting feature that the vortex density is height dependent. The apex is the region of low vorticity that coexists with the circular basis, the region with high vorticity. Figure 12 contains 25 figures, arranged in five columns and five rows, associated with the $(0, L)$ state. The left (magenta) column displays three-dimensional surface plots, which are isodensity plots (i.e., $|\psi|^{2}$ constant). In fact, each plot is made of two kinds of surfaces, namely, the inner ones which describe the vortices inside the cone and an outer one very near the surface of the cone. The following two columns of Fig. 12 are contour plots of the density and phase, respectively, taken at the circular basis $\left(z=z_{0}=4 \xi\right)$. The two last columns of Fig. 12, following the left to the right direction, are also contour plots of the density and phase, taken at two other cross section planes, namely, $z$ $=2 \xi$ and $z=\xi$, respectively. Figure 13 is associated with the $(1, L)$ state and is arranged in a similar fashion, but has just four columns and four rows. Top to bottom rows of Figs. 12 and 13 are in ascending magnetic field order, with the former and latter describing low and high fields, respectively. Figure 13 does not show the cross section plane $z=\xi$ plots because the superconducting state is nearly destroyed there by the presence of the central vortex, as seen in the threedimensional surface plots of its left column.

Although the same color scheme blue to red is used for all two-dimensional plots, its meaning is not the same for all of them, as it corresponds to different scales. For all the phase plots, the blue to red hue corresponds to the same scale of $0-2 \pi$. In the Cooper-pair density plots, the blue to red hue 

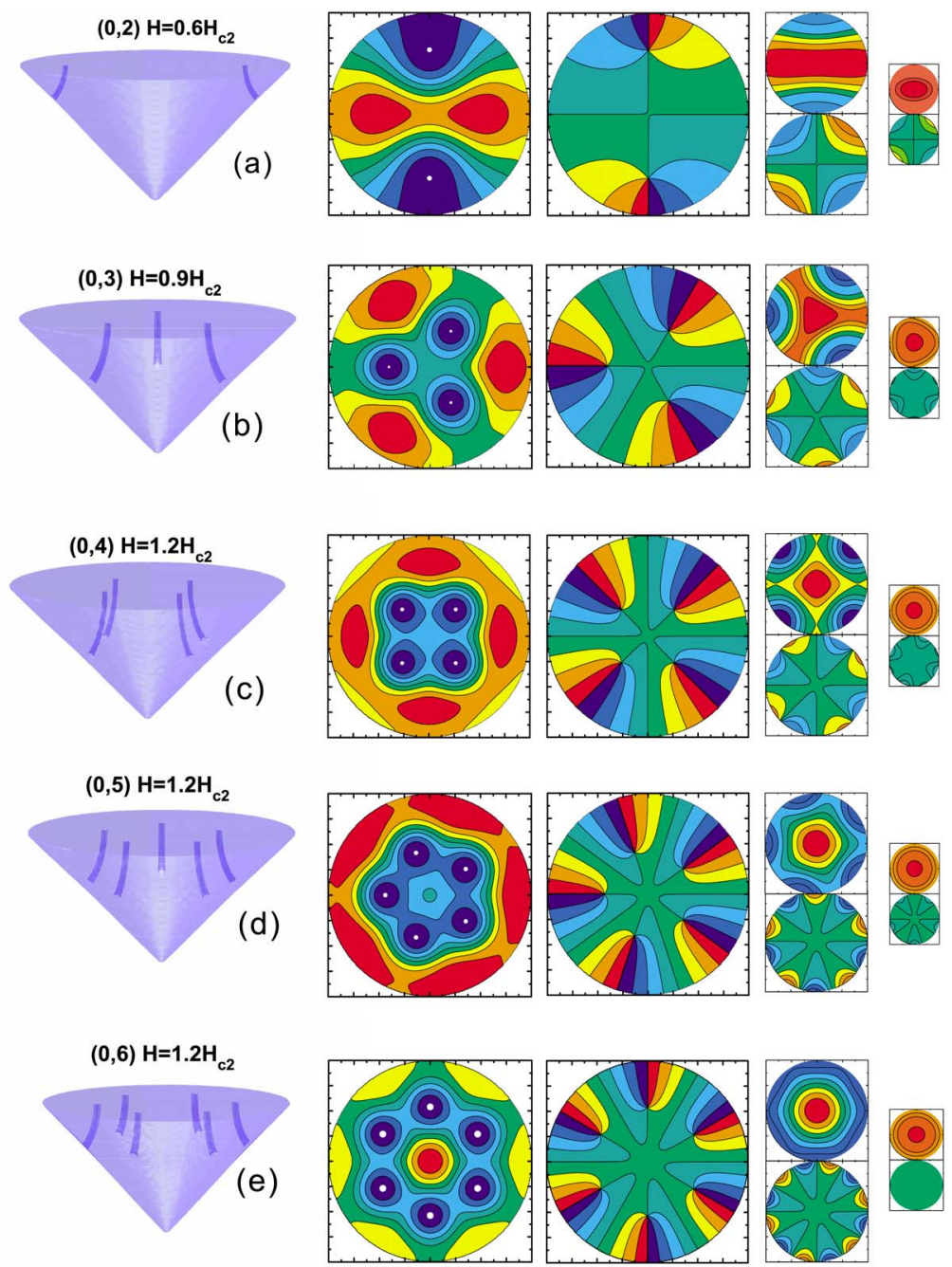

FIG. 12. (Color online) Plots of the vortex configuration, Cooper-pair density, and order parameter phase for the (meta-) stable $\operatorname{MVS}(0, L)$ $L=2-6$ at $H / H_{c 2}=0.6,0.9$, and 1.2 for the 4.0-45 cone. The Cooper-pair density and the order parameter phase are shown for selected planes: $z$ $=4 \xi, 2 \xi$, and $\xi$ (left to right). All these states are stable. (a) and (c) are the ground states, while the others are not. stands for minimum to maximum density, but the scale depends on the magnetic field value. For instance, above $H_{c 2}$, the order parameter starts to vanish, which means that the maximum density becomes very low although it is still being represented by the red color like it is for low fields. This is also true for the three-dimensional isodensity plots whose surface is drawn at some fraction of the maximum density. The white spots in two-dimensional isodensity plots stand for the region where the Cooper-pair density is lower than $10^{-3}$, except that in Fig. 13(d) which is lower than $10^{-4}$. Notice that both phase and density plots show very clearly the location of a vortex, by a border line between blue and red colors and by the white spots, respectively. To help the understanding, we provide in Table I the four different values of the density $|\psi|^{2}$ considered in Figs. 12-16.

To investigate the effects of the magnetic field into a $(0, L)$ vortex arrangement, the $\operatorname{MVS}(0,3)$ is considered at different fields $H / H_{c 2}=0.6,0.9$, and 1.2. Figure 14 displays the corresponding plots at these fields and shows that for increasing $H$, the vortices of the MVS $(0,3)$ shift toward the center. There is no vortex in the circular cone of Fig. 14(a) because the applied magnetic field is not sufficiently large for this. Notice that the three-dimensional isodensity plots of Fig. 14 are taken at different values, according to Table I.
Figure 15 shows the $(1,5)$ MVS at different fields, $H / H_{c 2}=1.06,1.13$, and 1.21 , respectively. It helps to understand the effects of the magnetic field on the $(1, L)$ kind of state. Again the three-dimensional isodensity plots of Fig. 15 are taken at different values according to Table I. The small white holes in the plots of Cooper-pair density in Fig. 15 mean that the Cooper-pair density there is smaller than 0.001 , but still different from zero. Here, we find that (i) when the applied magnetic field is not sufficiently large, there are no vortices away from the center and one vortex in the center with very small radius; (ii) with increasing magnetic field, vortices around the center appear; they will move toward the center and become bigger.

To complete our Cooper-pair and phase studies, we consider the 2.5-60 cone. Figure 16 shows the following MVS states: $(0,7) H / H_{c 2}=1.2,(1,7) \quad H / H_{c 2}=1.19$, and $(2,7)$ $H / H_{c 2}=1.21$. Figure 16 shows the $(0,7) \mathrm{MVS}$ at $H / H_{c 2}$ $=1.2$. Seven vortices are arranged on a shell around the center; the $(1,7) \mathrm{MVS}$ at $H / H_{c 2}=1.19$ has a shell with six vortices and a vortex in the center; the $(2,7) \mathrm{MVS}$ at $\mathrm{H} / \mathrm{H}_{c 2}$ $=1.21$ has vorticity 7 with five vortices on a shell and one GVS with vorticity 2 in the center.

Similar to the thin disk, the circular cone with size comparable to $\xi$ only supports GVS. Increasing its size allows for 


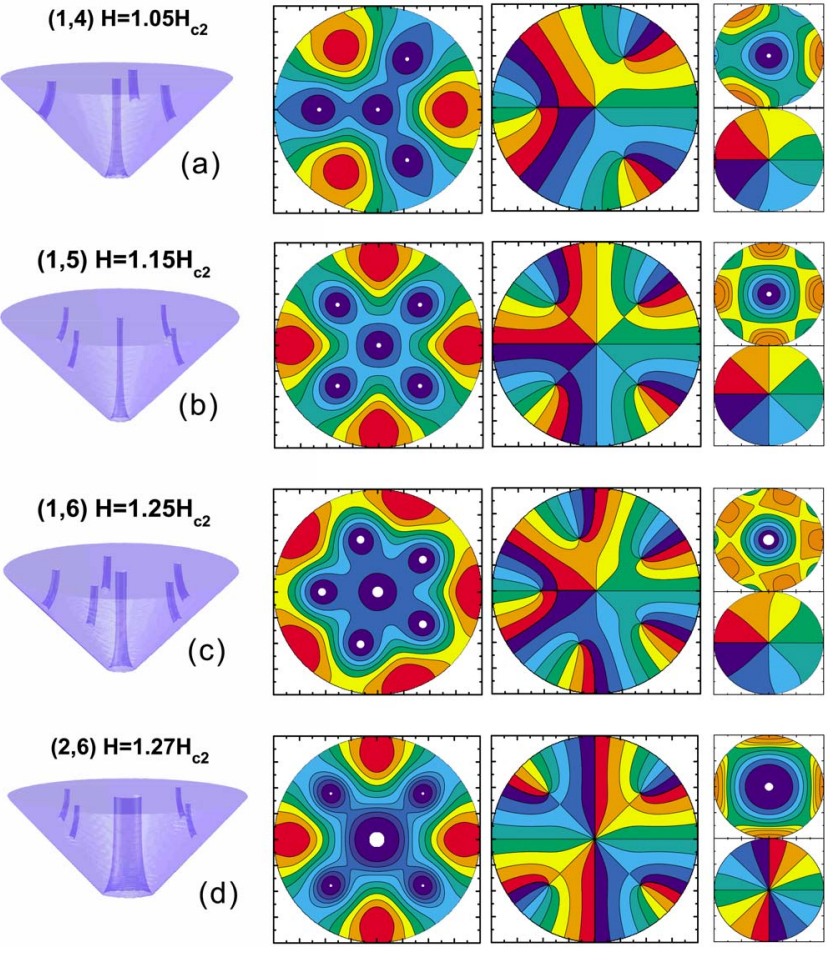

FIG. 13. (Color online) Plots of the vortex configuration, Cooper-pair density, and order parameter phase for the (meta-) stable MVS $(1, L) L=4-6$ and $(2,6)$ at $H / H_{c 2}=1.05,1.15,1.25$, and 1.27 for the 4.0-45 cone. The Cooper-pair density and the order parameter phase are shown for selected planes: $z=4 \xi$ and $2 \xi$ (left to right).

the onset of MVS described through Eq. (7), with coefficients given by Eq. (19). However, for the cone, the GVS always occupies its major axis and does not break into a MVS, as it does for the narrow constriction problem of Ref. 23.
TABLE I. Density values (arbitrary units) are shown for some figures: the three-dimensional isosurface density $\left(|\psi|_{3 D}^{2}\right)$, the density at the two-dimensional white holes $\left(|\psi|_{w h}^{2}\right)$, and the maximum density at the planes $z_{4} \equiv 4 \xi\left[|\psi|_{\max }^{2}\left(z_{4}\right)\right]$ and $z_{2} \equiv 2 \xi\left[|\psi|_{\max }^{2}\left(z_{2}\right)\right]$.

\begin{tabular}{lllll}
\hline \hline Figure & $|\psi|_{3 D}^{2}$ & $|\psi|_{w h}^{2}$ & $|\psi|_{\text {max }}^{2}\left(z_{4}\right)$ & $|\psi|_{\text {max }}^{2}\left(z_{2}\right)$ \\
\hline $12(\mathrm{a})$ & 0.001 & 0.001 & 0.76 & 0.888 \\
$12(\mathrm{~b})$ & 0.005 & 0.001 & 0.59 & 0.486 \\
$12(\mathrm{c})$ & 0.002 & 0.001 & 0.257 & 0.199 \\
$12(\mathrm{~d})$ & 0.002 & 0.001 & 0.227 & 0.215 \\
$12(\mathrm{e})$ & 0.001 & 0.001 & 0.0925 & 0.224 \\
$13(\mathrm{a})$ & 0.005 & 0.001 & 0.422 & 0.279 \\
$13(\mathrm{~b})$ & 0.002 & 0.001 & 0.269 & 0.1505 \\
$13(\mathrm{c})$ & 0.001 & 0.001 & 0.0766 & 0.0258 \\
$13(\mathrm{~d})$ & 0.005 & 0.001 & 0.0728 & 0.0264 \\
$14(\mathrm{a})$ & 0.05 & 0.05 & 0.744 & 1.048 \\
$14(\mathrm{~b})$ & 0.005 & 0.001 & 0.59 & 0.486 \\
$14(\mathrm{c})$ & 0.005 & 0.001 & 0.364 & 0.288 \\
$15(\mathrm{a})$ & 0.005 & 0.001 & 0.264 & 0.306 \\
$15(\mathrm{~b})$ & 0.002 & 0.001 & 0.256 & 0.186 \\
$15(\mathrm{c})$ & 0.001 & 0.001 & 0.23 & 0.036 \\
$16(\mathrm{a})$ & 0.0005 & 0.0005 & 0.097 & \\
$16(\mathrm{~b})$ & 0.0005 & 0.0005 & 0.0195 & \\
$16(\mathrm{c})$ & 0.0005 & 0.0005 & 0.0223 & \\
\hline \hline
\end{tabular}

\section{PHASE DIAGRAMS}

In this section, we obtain phase diagrams for the cone that well summarize the most important results. Figure 17 shows the apex angle versus the applied magnetic field for a few fixed heights, namely, $z_{0} / \xi=2.0,3.0$, and 4.0, respectively. According to Figs. 17(a), 17(c), and 17(e), the frontier between the normal region and the superconducting one is
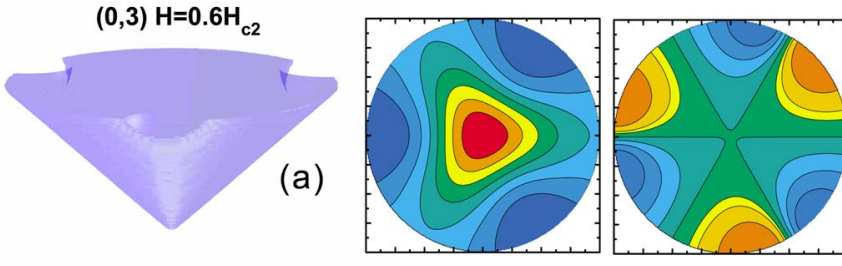

$(0,3) \mathrm{H}=0.9 \mathrm{H}_{\mathrm{c} 2}$
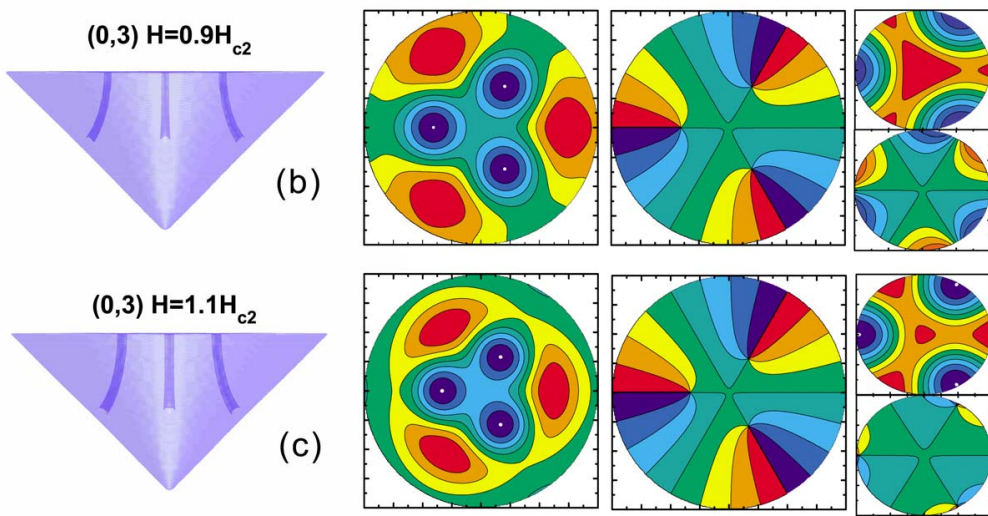
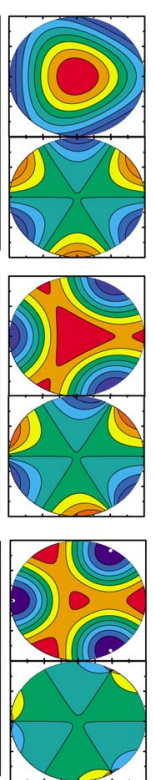

FIG. 14. (Color online) Plots of the vortex configuration, Cooper-pair density, and order parameter phase for the (meta-) stable MVS $(0,3)$ at $H / H_{c 2}=0.6,0.9$, and 1.1 for the 4.0-45 cone. The Cooper-pair density and the order parameter phase are shown for the $z=4 \xi$ and $z=2 \xi$ planes. 

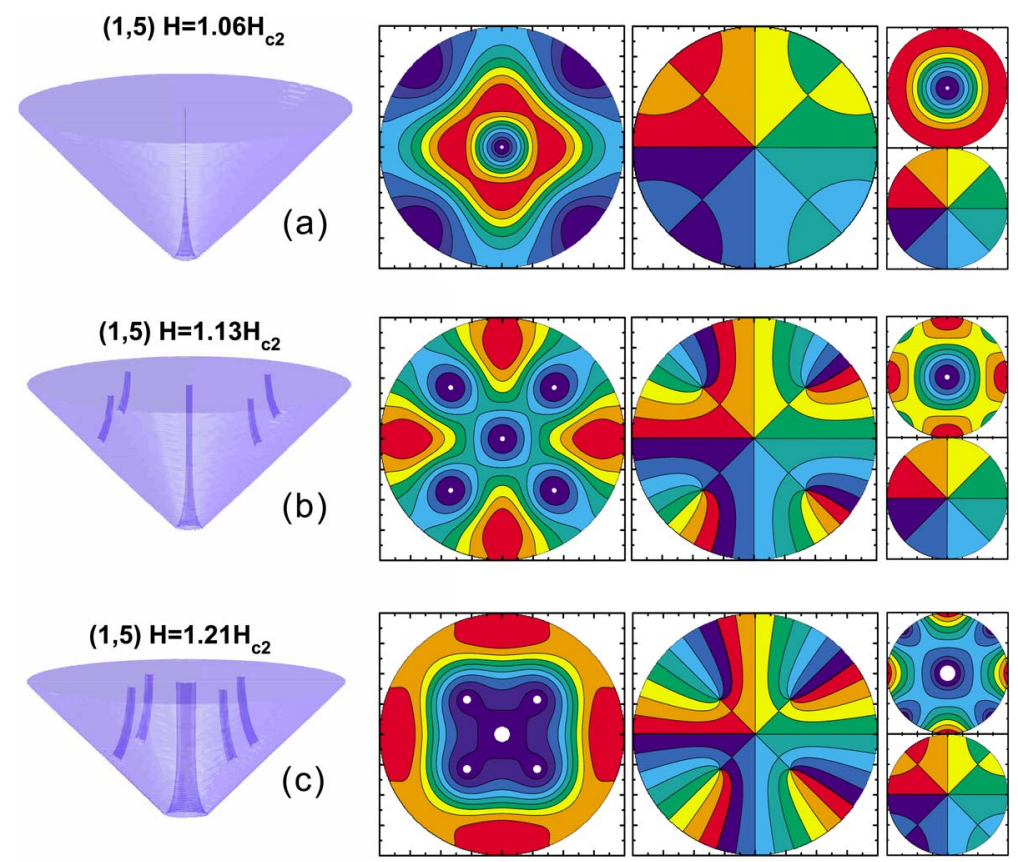

FIG. 15. (Color online) The plots of the vortex configuration, Cooper-pair density, and the phase of the order parameter of the MVS $(1,5)$ at the applied magnetic fields $H / H_{c 2}=1.06,1.13$, and 1.21 for the 4.0-45 cone. The Cooper-pair density and the order parameter phase are shown for the $z=4 \xi$ and $z=2 \xi$ planes. mostly covered by a state with no vortices $(L=0)$. An arrow points to the small region of this diagram that contains vortices $(L \neq 0)$. Extremely large fields can be reached in case of a very small apex angle in comparison to $H_{c 2}$. This supports the experimental observation that superconducting tips used in scanning tunneling spectroscopy can reach fields much higher than that of the bulk. ${ }^{18,21}$ For angles approximately above $55^{\circ}, 43^{\circ}$, and $32^{\circ}$, vortices can be present for the $z_{0} / \xi=2.0,3.0$, and 4.0 cones, respectively. Figures 17(b), $17(\mathrm{~d})$, and 17(f) give a zoom of the areas containing vortices. An integer number gives the maximum vorticity that can fit into each region. The (green) shading contains MVS, whereas the remaining (yellow) light region has GVS. A reentrant behavior is found for the two largest cones: $z_{0} / \xi$ $=3.0$ and 4.0. For an apex angle, approximately smaller than $47^{\circ}$ and $43^{\circ}$ in these two cases, respectively, it is possible by increasing the field to start and end without a vortex $(L=0)$ while in the superconducting state and still go through an intermediate MVS configuration.

Figure 18 shows the applied magnetic field versus temperature phase diagram, which contains the border line separating the superconducting to the normal state, for some selected cones. This figure also displays the stability lines for the GVS with vorticity $L$. Notice that, according to Fig. 17,
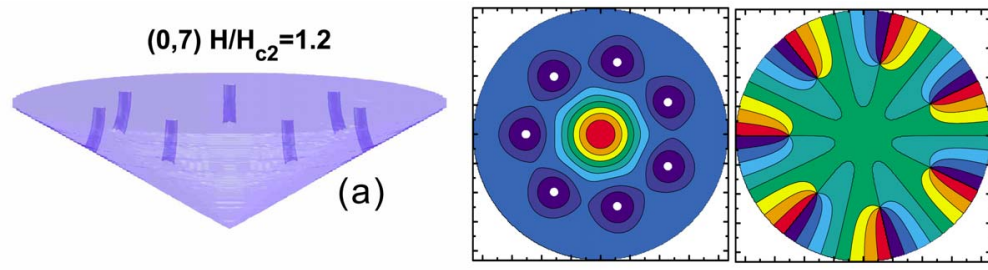

$(1,7) \mathrm{H} / \mathrm{H}_{\mathrm{c} 2}=1.19$
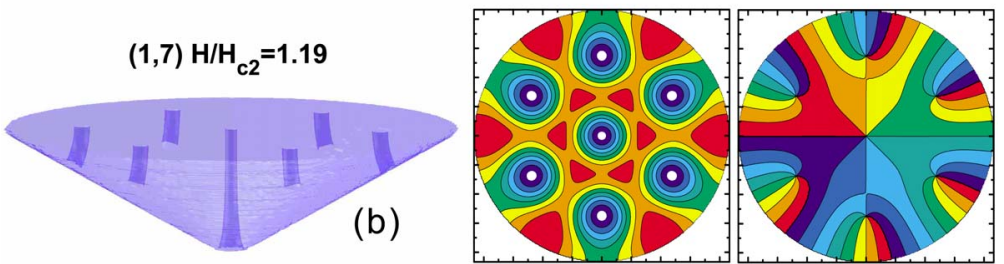

$(2,7) \mathrm{H} / \mathrm{H}_{\mathrm{c} 2}=1.21$
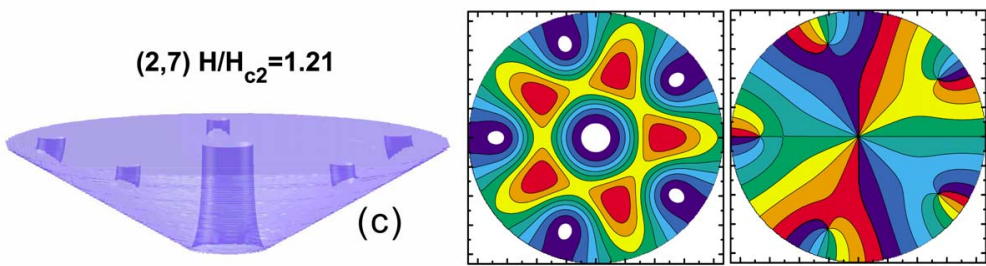

FIG. 16. (Color online) The plots of the vortex configuration, Cooper-pair density, and the phase of the order parameter of the MVS $(0,7)$, $(1,7)$, and $(2,7)$ at $H / H_{c 2}=1.2,1.19$, and 1.21 for the 2.5-60 cone. The Cooper-pair density and the order parameter phase are shown for the $z=4 \xi$ plane. The white hole stands for very low Cooper-pair density. 

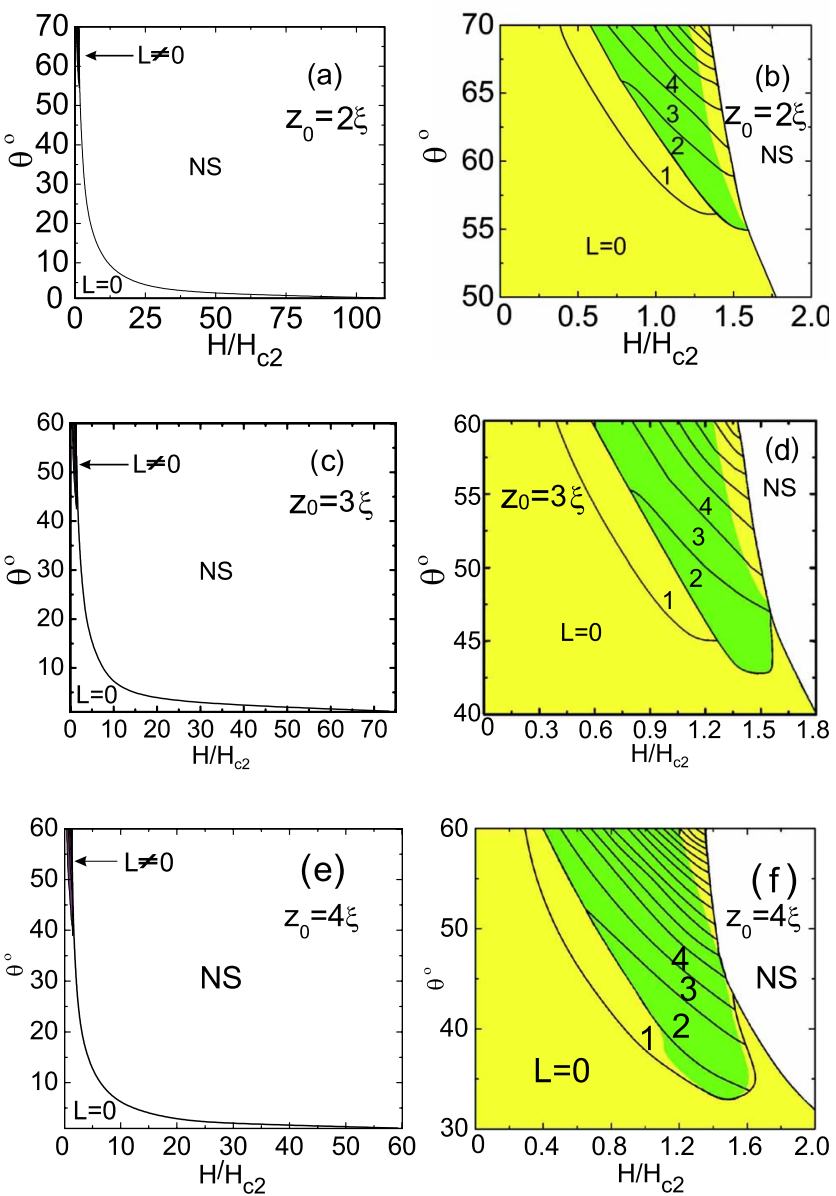

FIG. 17. (Color online) Apex angle versus applied field for the $z_{0} / \xi=2$ cone. According to (a), most of this phase diagram is a no vortex $(L=0)$ region. (b) is a zoom over the region that contains vortices $(L \neq 0)$. The (green) shaded region represents the MVS region, whereas the (yellow) light region has GVS. (c) and (d) are for the cone with $z_{0} / \xi=3$, while (e) and (f) are for the cone with $z_{0} / \xi=4$. near the border line where the superconducting state ceases to exist, there are basically just GVS.

Figure 17 helps to understand many properties of the cone. For instance, the $z_{0} / \xi=4$ with apex angle $\theta=38^{\circ}$ goes through two $L=1$ states by increasing the field. Consider the two possible magnetic field values, $H / H_{c 2}=1$ and $H / H_{c 2}$ $=1.15$. They fall, respectively, in the (green) shaded and in the (yellow) light regions, and, therefore, must correspond to a $(0,1)$ MVS and a $L=1 \mathrm{GVS}$, respectively. Notice that we have not discussed the MVS $(0,1)$ so far because it is not stable for the studied circular cones (2.5-45, 4.0-45, and 2.5$60)$. However, for the 4.0-38 cone, the $L=1 \mathrm{GVS}$ and the $(0,1) \mathrm{MVS}$ are the ground state at $H / H_{c 2}=1$ and $H / H_{c 2}$ $=1.15$, respectively. According to Fig. $17(\mathrm{e})$, the $(0,1)$ MVS corresponds to a single bent vortex, while the $L=1 \mathrm{GVS}$ takes the cone major axis, as seen in Fig. 19(b).

\section{CONCLUSION}

The vortex state of a mesoscopic superconducting circular cone was obtained using the Ginzburg-Landau theory. The applied magnetic field is perpendicular to the circular basis. The vortex density depends on the cone height because vortices emerge perpendicularly to the surface. Thus, the only vortex able to reach the cone apex is the one occupying the central axis. We solved the linear Ginzburg-Landau equation and expressed the order parameter in terms of its eigenfunctions with negative eigenvalues, the only ones useful to construct a solution of the nonlinear Ginzburg-Landau equation. Only two components are taken in this expansion and found to give an accurate description of giant and multivortex states. The two components are labeled by the azimuthal angular momentum pair $\left(L_{1}, L_{2}\right)$, where $L_{2}>L_{1}$. In the low field range, $L_{2}$ vortices form a shell around the center with no vortex in the center $\left(L_{1}=0\right)$. With increasing field, vortices move toward the center and, for sufficiently large field, $L_{2}$ $-L_{1}$ vortices can form a shell, while there is one giant vortex with vorticity equal to $L_{1}$ at the center. The present results
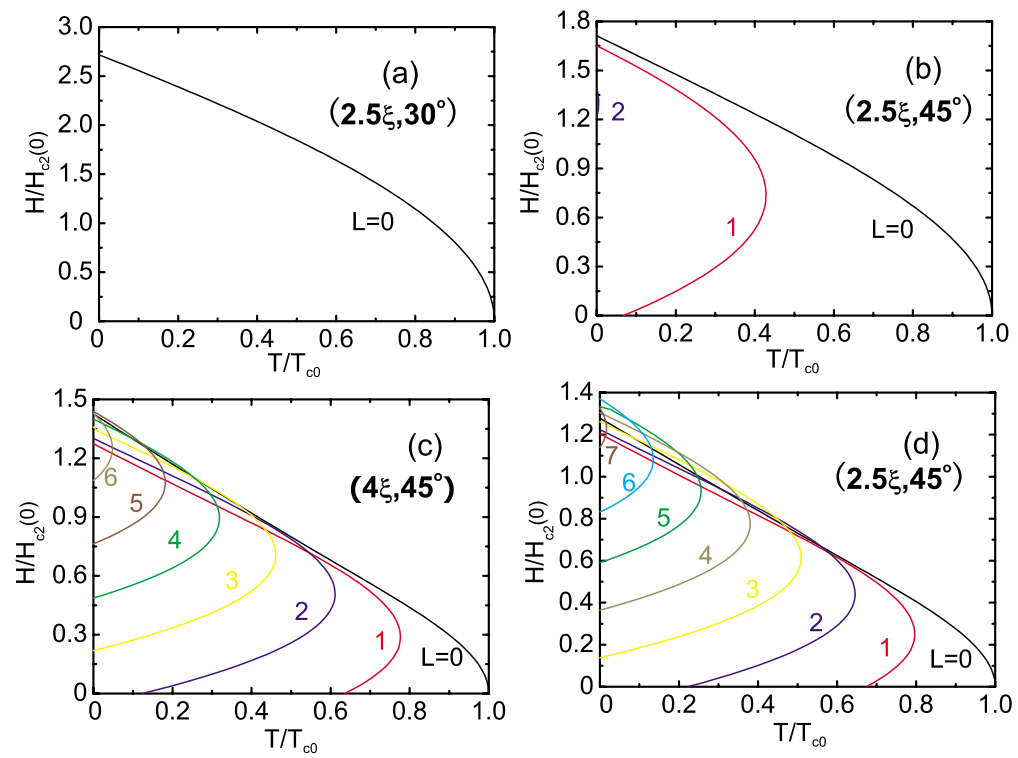

FIG. 18. (Color online) The phase diagram of applied field versus temperature is shown here for selected cones. GVSs with vorticity $L$ are shown near the superconducting-normal boundary. 

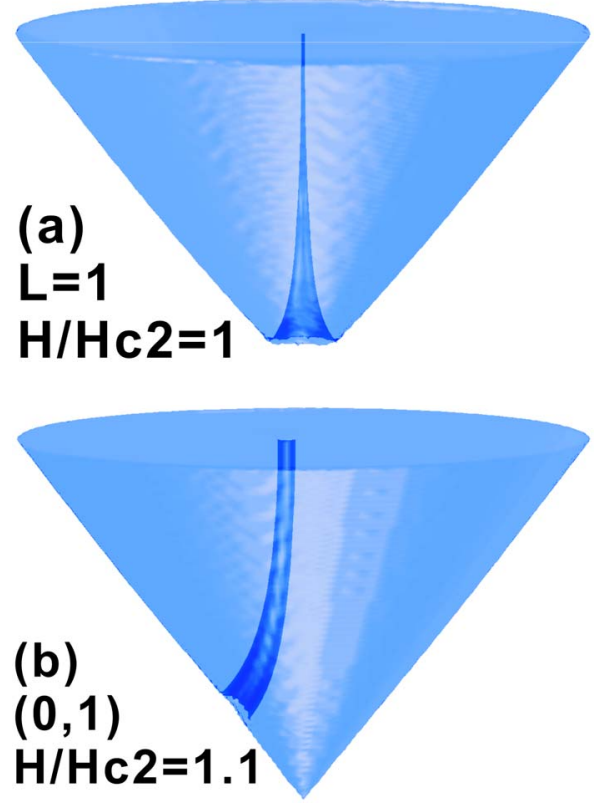

FIG. 19. (Color online) The 4.0-38 cone has two possible single vortex states, namely, (a) the $(0,1) \mathrm{MVS}$ at $H / H_{c 2}=1.15$ and (b) the $L=1 \mathrm{GVS}$ at $H / H_{c 2}=1$. The isosurfaces are drawn at densities (a) 0.005 and (b) 0.02 , as discussed in Table I.

contribute to the understanding of the superconducting properties of tips in the presence of an external magnetic field, which are currently used in scanning tunneling spectroscopy measurements.

\section{ACKNOWLEDGMENTS}

We thank Antonio R. de C. Romaguera, Arkady Shanenko, and Vyacheslav Misko for helpful discussions. M.M.D. thanks CNPq, FAPERJ, Instituto do Milênio de Nanotecnologia (Brazil), and BOF/UA (Belgium) for financial support. F.M.P. acknowledges support from the Flemish Science Foundation (FWO-Vl), the Belgian Science Policy (IAP), and the ESF-AQDJJ network.

\section{APPENDIX}

In the main text, we limited ourselves to eigenvalues of the linear problem, defined by Eq. (7), that have zero radial
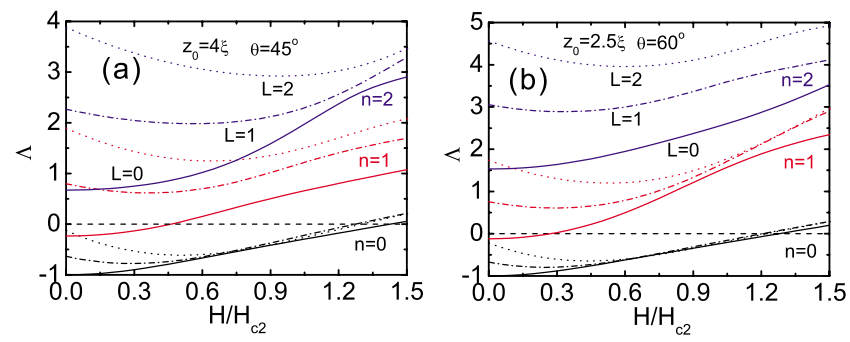

FIG. 20. (Color online) Eigenvalue $\Lambda_{L, n}$ as a function of $H / H_{c 2}$ are shown in (a) and (b) for the 4.0-45 and the 2.5-60 cones, respectively. States are represented in the following way: $L=0$ (solid curves), $L=1$ (dotted dashed curves), and $L=2$ (dotted curves); $n$ $=0$ (black curves), $n=1$ (red curves), and $n=2$ (blue curves). The dashed horizontal lines are just a guide for the eye $(\Lambda=0$ level).
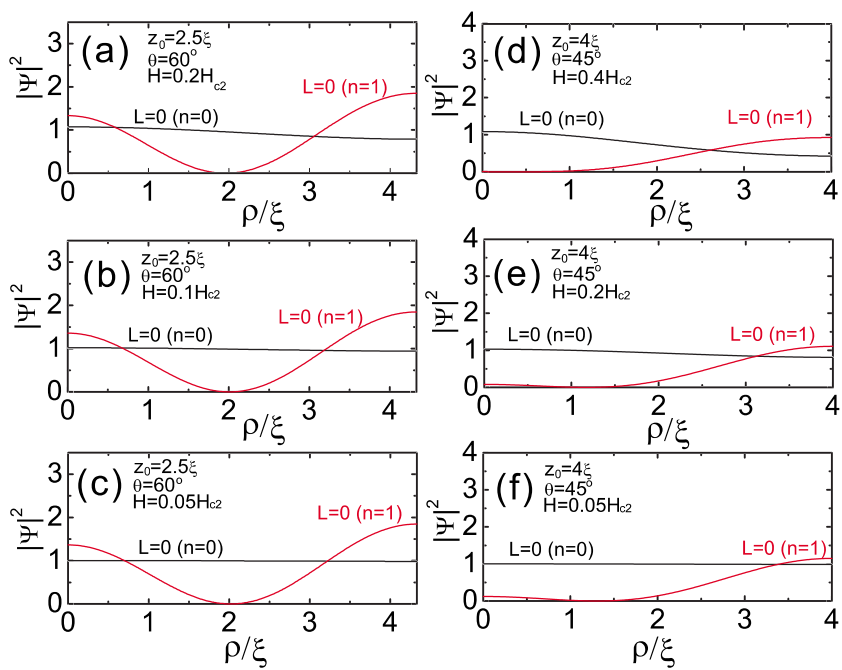

FIG. 21. (Color online) The radial dependence of the Cooperpair density for the $(L=0, n=0)$ and $(L=0, n=1)$ states are shown in the circular basis $\left(z=z_{0}\right)[(\mathrm{a})-(\mathrm{c})]$ for the 2.5-60 and [(d)-(f)] for the 4.0-45 cones at selected applied magnetic fields, chosen in the region that the $(L=0, n=1)$ state has a negative eigenvalue.

quantum number, i.e., $n=0$. Here, we investigate those states with $n \neq 0$ and refer to them as high- $n$ states. The corresponding eigenvalues $\Lambda_{L, n \neq 0}$ increase with $n$ for fixed $L$. A positive eigenvalue $\Lambda_{L, n}$ is not useful for the present purposes because only negative eigenvalue states can be a solution of the nonlinear GL theory. Most important, we find, beyond the present two-component framework, that the high$n$ states are unstable, although still falling in the negative eigenvalue regime.

Figures 20(a) and 20(b) show the first nine eigenvalue curves versus the applied magnetic field $\left(\Lambda_{L, n}, L=0,1,2\right.$ and $n=0,1,2)$ for the 4.0-45, and 2.5-60 cones, respectively. According to them, there is only one high- $n$ state with a negative eigenvalue regime, namely, the $(L=0, n=1)$ state. This happens within a limited window of low applied magnetic field.

Next, we plot the profiles of the Cooper-pair density for the $(L=0, n=0),(L=0, n=1)$ states along the radial direction of the circular plane basis (plane $z=z_{0}$ ). The two circular

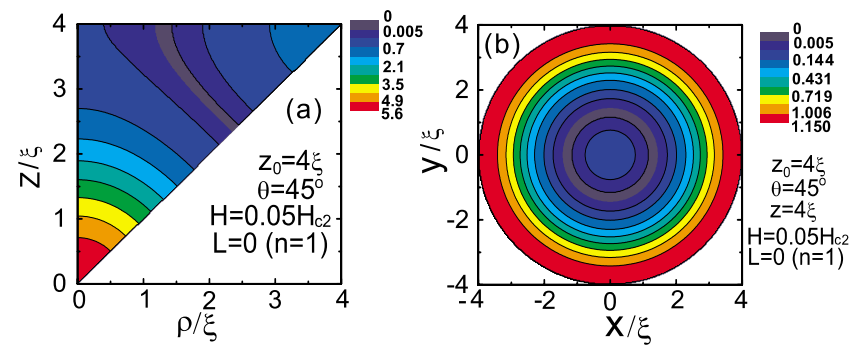

FIG. 22. (Color online) The Cooper-pair density of the first high- $n$ state, $(L=0, n=1)$, at $H / H_{c 2}=0.05$ for the 4.0-45 cone is shown for two cross section planes, namely, in the $\rho-z$ and $z=z_{0}$ planes. This last one is just a two-dimensional view of Fig. 21(f). Both plots have high Cooper-pair density in red, and low in blue, though they are at different scales. 
cones of Figs. 21(a) and 21(b) are considered at some selected $H$ values within the regime that the state $(L=0, n$ $=1$ ) has a negative eigenvalue, and therefore, interesting to construct the vortex state. From these figures, we find that the Cooper-pair density of the $(L=0, n=1)$ state vanishes within a ring at this surface, a feature already found in the thin disk problem (see Ref. 1), where one finds that it is not suppressed by increasing $H$ within the negative eigenvalue regime. Finally, we show in Fig. 22 the Cooper-pair density of state $(L=0, n=1)$ at $H / H_{c 2}=0.05$ for the $4.0-45$ cone in two cross section cuts, namely, along the $\rho-z$ and the $z=z_{0}$ planes. *francois.peeters@ua.ac.be

${ }^{1}$ S. V. Yampolskii and F. M. Peeters, Phys. Rev. B 62, 9663 (2000).

${ }^{2}$ V. A. Schweigert and F. M. Peeters, Phys. Rev. Lett. 83, 2409 (1999).

${ }^{3}$ R. Benoist and W. Zwerger, Z. Phys. B: Condens. Matter 103, 377 (1997)

${ }^{4}$ J. J. Palacios, Phys. Rev. B 58, R5948 (1998).

${ }^{5}$ P. S. Deo, V. A. Schweigert, F. M. Peeters, and A. K. Geim, Phys. Rev. Lett. 79, 4653 (1997).

${ }^{6}$ V. A. Schweigert and F. M. Peeters, Phys. Rev. B 57, 13817 (1998).

${ }^{7}$ A. Kanda, B. J. Baelus, F. M. Peeters, K. Kadowaki, and Y. Ootuka, Phys. Rev. Lett. 93, 257002 (2004).

${ }^{8}$ B. J. Baelus, S. V. Yampolskii, F. M. Peeters, E. Montevecchi, and J. O. Indekeu, Phys. Rev. B 65, 024510 (2001).

${ }^{9}$ A. R. de C. Romaguera, M. M. Doria, and F. M. Peeters, Phys. Rev. B 75, 184525 (2007).

${ }^{10}$ B. J. Baelus, S. V. Yampolskii, and F. M. Peeters, Phys. Rev. B 66, 024517 (2002).

${ }^{11}$ Guo-Qiao Zha, Shi-Ping Zhou, Bao-He Zhu, Yao-Ming Shi, and Hong-Wei Zhao, Phys. Rev. B 74, 024527 (2006).

${ }^{12}$ M. M. Doria, A. R. de C. Romaguera, and F. M. Peeters, Phys. Rev. B 75, 064505 (2007).

${ }^{13}$ B. J. Baelus, D. Sun, and F. M. Peeters, Phys. Rev. B 75, 174523 (2007).

${ }^{14}$ A. R. de C. Romaguera, M. M. Doria, and F. M. Peeters, Phys. Rev. B 76, 020505(R) (2007).

${ }^{15}$ V. A. Schweigert, F. M. Peeters, and P. S. Deo, Phys. Rev. Lett. 81, 2783 (1998).

${ }^{16}$ J. G. Rodrigo, H. Suderow, and S. Vieira, Eur. Phys. J. B 40, 483 (2004).

${ }^{17}$ S. Pan, E. Hudson, and J. Davis, Appl. Phys. Lett. 73, 2992 (1998).
${ }^{18}$ H. Suderow, E. Bascones, A. Izquierdo, F. Guinea, and S. Vieira, Phys. Rev. B 65, 100519(R) (2002).

${ }^{19}$ A. Kohen, T. Proslier, T. Cren, Y. Noat, W. Sacks, H. Berger, and D. Roditchev, Phys. Rev. Lett. 97, 027001 (2006).

${ }^{20}$ M. Poza, E. Bascones, J. G. Rodrigo, N. Agraït, S. Vieira, and F. Guinea, Phys. Rev. B 58, 11173 (1998).

${ }^{21}$ J. Rodrigo, H. Suderow, and S. Vieira, Phys. Status Solidi B 237, 386 (2003).

${ }^{22}$ V. R. Misko, V. M. Fomin, and J. T. Devreese, Phys. Rev. B 64, 014517 (2001).

${ }^{23}$ A. K. Elmurodov, D. Y. Vodolazov, and F. M. Peeters, Europhys. Lett. 74, 151 (2006).

${ }^{24}$ B. J. Baelus, F. M. Peeters, and V. A. Schweigert, Phys. Rev. B 63, 144517 (2001).

${ }^{25}$ A. K. Geim, I. V. Grigorieva, S. V. Dubonos, J. G. S. Lok, J. C. Maan, A. E. Filippov, and F. M. Peeters, Nature (London) 390, 259 (1997).

${ }^{26}$ A. K. Geim, S. V. Dubonos, I. V. Grigorieva, K. S. Novoselov, F. M. Peeters, and V. A. Schweigert, Nature (London) 407, 55 (2000).

${ }^{27}$ I. V. Grigorieva, W. Escoffier, J. Richardson, L. Y. Vinnikov, S. Dubonos, and V. Oboznov, Phys. Rev. Lett. 96, 077005 (2006).

${ }^{28}$ I. V. Grigorieva, W. Escoffier, V. R. Misko, B. J. Baelus, F. M. Peeters, L. Y. Vinnikov, and S. V. Dubonos, Phys. Rev. Lett. 99, 147003 (2007).

${ }^{29}$ Liviu F. Chibotaru, Arnout Ceulemans, Vital Bruyndoncx, and Victor V. Moshchalkov, Nature (London) 408, 833 (2000).

${ }^{30}$ R. Geurts, M. V. Milosevic, and F. M. Peeters, Phys. Rev. Lett. 97, 137002 (2006).

${ }^{31}$ M. Tinkham, Introduction to Superconductivity (McGraw-Hill, New York, 1975).

${ }^{32}$ V. A. Schweigert and F. M. Peeters, Phys. Rev. B 60, 3084 (1999). 